\title{
BAND STRUCTURE EFFECTS IN THE FIELD ELECTRON EMISSION FROM HTSC MONOCRYSTALS
}

\author{
I.V. Uimanov \\ Institute of Electrophysics, 106 Amundsen St., Ekaterinburg 620016, Russia \\ FAX: +7 (3432) 678694, uimanov@,iep.uran.ru
}

PACS: 79.70.+q; 74.72.-h

Keywords: field emission; High- $T_{c}$ compounds; superconductors

\begin{abstract}
This paper presents a theoretical approach developed for describing field-emission characteristics of type $\mathrm{YBa}_{2} \mathrm{Cu}_{3} \mathrm{O}_{7-\mathrm{y}}$ copper-oxide high- $T_{\mathrm{c}}$ superconductors in the normal state. The approach developed is based on taking into account the topological features of the essentially anisotropic band structure of the given compounds in the strong bond approximation and the bending of the energy bands under the conditions of the field-emission experiment because of the low concentration of mobile charge carriers. Approximate analytic expressions have been derived and numerical calculations of the field-emission spectra in the [100] and [010] directions have been performed. Based on the results obtained, the principal "abnormal" fieldemission characteristics of $\mathrm{YBa}_{2} \mathrm{Cu}_{3} \mathrm{O}_{7-y}$ for the (100) plane, observed experimentally, have been explained. It has been shown that detailed field-emission-spectroscopy data for type $\mathrm{YBa}_{2} \mathrm{Cu}_{3} \mathrm{O}_{7-\mathrm{y}} \mathrm{HTSC}$ emitters make it possible to investigate the main parameters of the band structure of these compounds.
\end{abstract}




\section{INTRODUCTION}

The nature of normal (non-superconductive) state has thus far been one of the significant aspects of HTSC problem in copper-oxide compounds. While in traditional metal superconductors the pattern of Fermi-liquid with a well-distinguished spectrum of quasi-particles near the Fermi surface has been established rather rigorously, in copper-oxide compounds, however, this issue remains debatable due to strong Coulomb correlations. It is generally recognized that an in-detail investigation into the physical pattern of the normal state can well serve as a key to the HTSC theory advancement. Therefore, studying the normal state of copperoxide high- $T_{\mathrm{c}}$ superconductors remains quite important. Moreover, the intense interest focused in this area of study has caused a number of 'abnormal' properties to be revealed in the normal state. As that has taken place, it has become obvious that, unlike traditional superconductors with their superconductivity being metal-based, the copper-oxide compounds possess a variety of unusual 'nonmetallic' physical properties. It is also worthy to note that many features of electronic composition of such compounds can be investigated in the normal state already.

Efforts to experimentally investigate the HTSC phenomenon by means of field ion microscopy (FIM), field electron spectroscopy (FES) and microscopy (FEEM) started almost immediately upon the discovery of superconductivity in cuprates [1]. The experience accumulated in previous work by N.N. Syutkin at.al. [2,3], G. Kellog at. al. [4,5] and A. Melmed at. al. $[6,7]$ has made it possible to successfully utilize field ion microscopy as part of the general test program to investigate the HTSC phenomenon by emission techniques. The next significant achievement in investigating HTSC materials by emission techniques was the application of ultrahigh vacuum field evaporation cleaning onto the emitter surface. In [8], the field emission spectra of $\mathrm{YBa}_{2} \mathrm{Cu}_{3} \mathrm{O}_{6.9}$ ceramics crystallites have for the first time been investigated after the field evaporation cleaning. Further investigations $[9,10]$ have resulted in stable and reproducible field emission spectra of $\mathrm{YBa}_{2} \mathrm{Cu}_{3} \mathrm{O}_{6.9}$. Further on, there have been investigations of $\mathrm{YBa}_{2} \mathrm{Cu}_{3} \mathrm{O}_{6.9}$ monocrystals by emission techniques based on a modified method for preparing 
point emitters [11-14]. These investigation made it possible to obtain field emission images of the cleaned surface of $\mathrm{YBa}_{2} \mathrm{Cu}_{3} \mathrm{O}_{7-\mathrm{y}}$ monocrystals with various contents of oxygen within a wide range of current density and at various temperatures. During these investigations it has also been proved that current-voltage characteristics of the given HTSC are straight lines in the FowlerNordheim coordinate frame, and the emission spectra, while showing some 'metallic' features, at the same time are characterized by such features as can be intrinsic to these materials only. In terms of the field electron emission theory for traditional metals, the following emission properties of $\mathrm{YBa}_{2} \mathrm{Cu}_{3} \mathrm{O}_{6.9}$ can be regarded as 'abnormal': the great width of high energy edge of emission spectrum $\sim 0.4 \mathrm{eV}$ at a temperature of $115-300 \mathrm{~K}$, the great total width of the emission spectrum $\sim 2 \mathrm{eV}$ at normal values of the electric field, for which the total width of emission spectrum $\mathrm{W} \sim 1 \mathrm{eV}$, the maximum of spectra is shifted by $0.2-1.5 \mathrm{eV}$ with regard to the spectrum maximum $\mathrm{W}$, and as the electric field strength grows, there is a shift in the spectrum observed.

In terms of the field emission theory, copper-oxide compounds are rather complicated objects, and the author does not know of any theoretical approach that would reach out of the scope of the isotropic Fermi gas approximation or out of the "isotropic metal BCS-like model" for that matter. On the other hand, analyzing major features of electron composition of such materials, one can reveal the basic properties, which should necessarily be taken into account. First of all, it is the strong anisotropy of electron properties, which, to a great extent, is of quasitwo-dimensional nature; secondly, it is the small concentration of mobile carriers, which dictate the necessity of adding the theory with screening effects of the external electric field, topographic features of band structure, etc. When considering an electron system for these compounds, it should be noted that the free electron approximation is inapplicable here, which rather complicates the theory and leads to the requirement to use, at least, simplified models in the strong bond approximation. 
Therefore, development of HTSC field emission theory, in our opinion, shows the real promise in the theoretical approaches described in $[15,16]$, which address investigations of the effects of band structure of transitional metals and semiconductors on their emission properties.

\section{FIELD EMISSION SPECTROSCOPY OF $\mathrm{YBa}_{2} \mathrm{Cu}_{3} \mathrm{O}_{7-\mathrm{y}}$ IN THE NORMAL STATE}

Let us basically outline an approach to the description of field emission characteristics of HTSC compounds and its difference from the field emission theory for traditional metals. Such approach implies the application of general expression for the barrier-transmission probability (without simplifications related to isotropy of the law of dispersion for mobile charge carriers), the allowance for the electric field penetrating into the emitter [17], and due consideration to the effects of band structure of HTSC's. We are to operate within a model, which represents the emitter surface as a crystallographic plane and the potential energy of electron at the emitter surface in vacuum depends only on the coordinate normal to the barrier. Within the framework of such model it has been shown in [15] that the barrier-transmission probability depends only on the electron energy in vacuum, which is normal to the barrier. Provided that the tangential component of momentum and total energy of electron remain the same during barrier tunneling, that results in the fact that the transmission coefficient for the barrier is described by a function $\varepsilon_{\perp}=\varepsilon_{\mathbf{k}}-\left(\hbar k_{t}\right)^{2} /(2 m)$, where $k_{t}$ is the electron quasi-momentum tangential to the barrier in the HTSC band, $\varepsilon_{\mathbf{k}}$ is the total electron energy in the HTSC band in a $\boldsymbol{k}$ state calculated from the bottom of band. Within the WKB approximation, the general expression for the transparence coefficient in case of under-barrier state takes the form $D\left(\varepsilon_{\perp}\right)=\left(1+\exp \left[Q\left(\varepsilon_{\perp}\right)\right]\right)^{-1}$ at $\varepsilon_{\perp} \geq 0$ and $D\left(\varepsilon_{\perp}\right)=0$ at $\varepsilon_{\perp}<0$. The complete expression for $D$ can be found in [18].

In common with many other approaches (see for example $[19,17]$ ), the band structure of HTSC we can take for a square lattice of dimension $a$. Penetration of the electric field results in 
the fact that, according to [17], the original spectrum of quasi-particles near the emission surface in the HTSC emitter takes the form

$$
E_{\mathbf{k}}(\boldsymbol{k})=\varepsilon_{k}-\delta R=-2 t\left(\cos \left(k_{a} a\right)+\cos \left(k_{b} a\right)\right)-4 t^{\prime} \cos \left(k_{a} a\right) \cos \left(k_{b} a\right)-\mu-\delta R,
$$

where $E_{\mathbf{k}}$ is the energy of itinerant electrons measured from the Fermi level, $\delta R$ is the value of the bending of band on the emission surface (we assume it to be positive). The energy, according to (1), should be calculated from the level of electrochemical potential $\mu_{\mathrm{ec}}$. Its value, when calculated from the bottom of band on the emission surface, amounts to $\mu_{\mathrm{ec}}=\varepsilon_{\mathrm{F}}+\delta R$, where $\varepsilon_{F}=4 t+4 t^{\prime}+\mu$ is the Fermi energy of the initial (unperturbed by electrical field) electron spectrum, calculated from the bottom of emitter's band. As this takes place, the electron distribution function near the emission surface will take the form $n_{\mathbf{k}}=\left(1+\exp \left[E_{\mathbf{k}} /\left(k_{B} T\right)\right]\right)^{-1}(\operatorname{see}$ Fig. 1).

Formal expression for the emission spectrum can be obtained within the framework of the tunnel Hamiltonian method and be represented as [20,21]:

$$
J\left(E_{q}\right)=2 e \iiint_{E_{q}, E_{q}+d E_{q}} A\left(\boldsymbol{k}, E_{q}\right) n_{\mathrm{FD}}\left(E_{q}\right) D\left(\varepsilon_{\perp}\right) \frac{1}{\hbar} \frac{\partial E_{\mathbf{k}}}{\partial k_{\perp}} \frac{d^{3} k}{(2 \pi)^{3}} .
$$

Where $A\left(\boldsymbol{k}, E_{q}\right)$ is the spectral density, $E_{q}$ is the total energy of emitted electrons calculated from the emitter's electrochemical potential level.

For the purposes of further calculation, the Eq. (2) is convenient to be transformed by applying integration over the constant energy surface projection $d s$ onto a plane parallel to the emission surface. Fig. 2 shows this area as shadowed. A more detailed description of this method can be found in [16]. Then, for the Eq. (2) we have:

$$
J\left(E_{q}\right)=\frac{2 e}{\hbar(2 \pi)^{3}} \int_{E_{q}, E_{q}+d E_{q}} n_{\mathrm{FD}}\left(E_{q}\right) d E_{\mathbf{k}} \iint A\left(\boldsymbol{k}, E_{q}\right) D\left(\varepsilon_{\perp}\right) d s .
$$

The expression (3) makes it possible not only to investigate the influence of band structure effects on the emission characteristics in the non-interacting system but also to consider various correlation phenomena. At present, there are quite a number of various theories that would, one way or another, address correlations in the electron system of HTSC. As a rule, such 
studies are aimed at providing an explanation either for the superconductivity phenomenon per se or for the pseudo-gap anomalies or some other abnormal characteristics of HTSC, which would have a characteristic energy scale of a few tens of meV. Our concern, however, is mainly in the 'emission anomalies' with the characteristic energy scale of a few hundred meV (see Introduction). Therefore, it is reasonable to consider the formation of emission spectra without correlations at first. Based on such assumption, the expression for the spectral density will take the form of a delta-function $A\left(\boldsymbol{k}, E_{q}\right) \rightarrow \delta\left(E_{\mathbf{k}}-E_{q}\right)$, and (3) will take the form:

$$
J\left(E_{q}\right)=\frac{2 e}{\hbar(2 \pi)^{3}} n_{\mathrm{FD}}\left(E_{q}\right) \iint D\left(\varepsilon_{\perp}\right) d s .
$$

It is known that for field emission the barrier-transmission coefficient $D<<1$. Then, applying the routine simplifying procedure to the barrier-transmission coefficient [18], we shall have the following:

$$
D\left(\varepsilon_{\perp}\right) \approx \begin{cases}\exp \left[-c_{E}-\frac{1}{d_{E}} \frac{\hbar^{2} k_{t}^{2}}{2 m}\right], & E_{q}+\mu_{\mathrm{ec}} \geq \frac{\hbar^{2} k_{t}^{2}}{2 m} \\ 0, & E_{q}+\mu_{\mathrm{ec}}<\frac{\hbar^{2} k_{t}^{2}}{2 m}\end{cases}
$$

where

$$
\begin{gathered}
c_{E}=\frac{4}{3}\left(\frac{2 m}{\hbar^{2}}\right)^{1 / 2} \frac{\left(\varphi-E_{q}\right)^{3 / 2}}{e F} v\left(y_{E}\right), \\
\frac{1}{d_{E}}=2\left(\frac{2 m}{\hbar^{2}}\right)^{1 / 2} \frac{\left(\varphi-E_{q}\right)^{1 / 2}}{e F} t\left(y_{E}\right), \\
t\left(y_{E}\right)=v\left(y_{E}\right)-(2 / 3) y_{E} d v / y_{E}, \quad y_{E}=\rho\left(e^{3} F\right)^{1 / 2} /\left(\varphi-E_{q}\right) .
\end{gathered}
$$

Here, $F$ is the electrical field strength, $\rho^{2}=(\varepsilon-1) /(\varepsilon+1), \varepsilon$ is the permittivity, and $t\left(y_{E}\right)$, $v\left(y_{E}\right)$ are the tabulated Nordheim functions [18].

With due consideration given to the band structure effects, further analysis of field emission spectra formation would be possible only if there were given relative orientation of crystallographic axis and the emission surface. 
Let us note that the majority of $\mathrm{YBa}_{2} \mathrm{Cu}_{3} \mathrm{O}_{7-\mathrm{y}}$ emission spectroscopy experimental results [11-14] have been obtained by probing the emission current from the (100) (or (010)) plane. That is why the [100] direction is of our greatest interest.

Let the normal to the emission surface coincide with a-axis (or b-axis). The relative orientation of the constant energy surface and the potential barrier at the boundary of HTSC emitter/vacuum is shown in Fig. 3. Then, the projections of constant energy surfaces, tangential to the emission direction, will be represented as rectangle areas of two types (shaded areas in Fig. 4. a), b). At $E_{q}<E^{\mathrm{VH}}$ these projections take the form of a simply connected rectangle domain centered on the point of origin (see Fig. 4. a). The energy $E^{\mathrm{VH}}=4 t^{\prime}-\mu-\delta R$ is the energy, at which the constant energy surfaces are open in the [100] direction. It is at the same energy that a van Hove singularity occurs in the density of state. At $E_{q}>E^{\mathrm{VH}}$, the tangential projections are two disconnected rectangle domains (see Fig. 4. b)). Analysis of nonzero barriertransmission probability Eq. (5) has shown that only the domain bounded by maximum $k_{t}^{\max }$ for each $E_{q}$ makes contribution into the integral over the tangential projection of constant energy. Fig. 4 shows this domain schematically as shaded. Physically, this bound is associated with the fact that electrons in the state with a quasi-momentum tangential projection, which reaches beyond the shaded domain, can not make contribution into the emission process. For such states the tangential energy of electron in vacuum $\left(\hbar k_{t}\right)^{2} /(2 m)$ should be greater than its total energy $\varepsilon_{k}$, which contradicts to the electronic selection rule by total energy. Therefore, the condition under consideration distinguishes a surface in the $\boldsymbol{k}$-space such that the barrier-transmission probability is nonzero only for the states within this surface. The Equation for this surface takes the form: $\varepsilon_{k}-\hbar^{2} k_{t}^{2} /(2 m)=0$. And the shape of this surface would depend on both the topographical features of band structure and the orientation to the emission surface. Fig. 5 shows this surface for the direction [100] under investigation and for the utilized model band of $\mathrm{YBa}_{2} \mathrm{Cu}_{3} \mathrm{O}_{7}$. 


\section{RESULTS AND DISCUSSION}

Figure 6 a) shows the emission spectra of a $\mathrm{YBa}_{2} \mathrm{Cu}_{3} \mathrm{O}_{6.9}$ emitter in the [100] direction obtained by means of numerical integration of Eq. (4) for the a of fields of $(3 \div 3.5) \cdot 10^{7} \mathrm{~V} / \mathrm{cm}$, which corresponds to a $17 \%$ increase in the anode voltage in the experiment. The corresponding dependence of a value of the bending of band is shown in Fig. 6 b). Let us consider the features of these spectra. At small $F$ the bending of band, which is due to the field penetration, is such as the electrochemical potential falls within the band (see Fig. 1). Accordingly, the high energy edge of the spectrum, or a part of it, is formed by the energy distribution function for electrons thus creating a 'Fermi edge' at $E_{q}=0$. As the strength of electrical field grows, the top of band goes down and at $\delta R>\varepsilon_{\max }$, the whole band near the emission surface is filled with electrons. Here $\varepsilon_{\max } \equiv \varepsilon_{\mathbf{k}}\left(k_{\mathrm{x}}=\pi / a, k_{\mathrm{y}}=\pi / a\right)=4 t-4 t^{\prime}-\mu$ is maximum band energy. As this occurs, the thermodynamic distribution by no mean affects the forming of spectrum. The shape of spectrum in this case is completely determined by the band structure. Topographical features of band structure are such as the exposure of the constant energy surfaces in the [100] direction results in a relative smoothing of the barrier-transmission probability for all band states. Thus electrons, which are in the states with the greatest total energy, have quite large tangential projections of a quasi-momentum $k_{t} \approx \pi / a$. Therefore, unlike the spherically symmetrical band structure typical for metals, the range of emitting states is not determined by the small vicinity of Fermi energy but is distributed practically over the whole band of mobile charge carriers. As this is taking place, the maximum of spectrum is formed by electrons which are not characterized by the highest energy. It should be pointed out that the approach developed in this paper makes it possible to explain the absence of 'Fermi edge' on the experimental spectra without a notion of destruction of Fermi surface by some strong correlations. However, this issue is of fundamental importance in order to establish the nature of the normal state in cuprates. The absence of the 
Fermi surface may results in the conclusion that the Fermi liquid approach is inadequate to the description of the electron system of copper-oxide, which thus far has been debatable [22].

A fairly good agreement should be noted between the results obtained and the experimental results described in [23]; the results agree both in the shape of spectrum and in the value of the spectral shift. Fig. $6 \mathrm{c}$ ) shows the calculation results for the width of the high energy edge of the emission spectrum $\Delta T$ (see Fig. 7). It is seen that $\Delta T$ amounts to $\sim 0.4 \mathrm{eV}$, which is in accordance with the experimental data $(0.32 \div 0.38 \mathrm{eV}$ [23]). Fig. 8 a) shows experimentally obtained function $\Delta S$ - the shift of the spectrum of $\mathrm{YBa}_{2} \mathrm{Cu}_{3} \mathrm{O}_{6.9}$ emitter relative to the Fermi level for a tungsten emitter [23]. The level of 0.67 of the spectrum maximum was used as a reference point for the spectrum on the energy scale (see Fig. 7). Assuming the screening length as unknown for this problem and using the data [23] on electrical field calibration, we made an adjusting in order to calculate $l_{\mathrm{TF}}$ [17]. The best result, which corresponded with the experimental data, was obtained at $l_{\mathrm{TF}}=3.4 \AA$. The calculated theoretical function $\Delta S$ is shown in Fig. 8 b). It should be noted that such value of $l_{\mathrm{TF}}$ is in agreement with the results obtained in research to investigate the effect of electrical field in $\mathrm{YBa}_{2} \mathrm{Cu}_{3} \mathrm{O}_{6.9}$ in planar structures (see [17] for more details). However, the most important is the fact that the approach thus developed has made it possible to utilize emission methods for investigation of screening parameters. The author of this paper does not know of alternative direct experimental techniques, which would establish the specific scope of electrical field penetration into cuprates.

Analysis of nonzero barrier-transmission probability has made it possible to obtain approximate analytical expression for emission spectrum of a $\mathrm{YBa}_{2} \mathrm{Cu}_{3} \mathrm{O}_{6.9}$ emitter $J_{[100]}\left(E_{q}\right)$ in the [100] (or [010]) direction, which can be presented in as follows: 


$$
\begin{aligned}
& J_{[100]}\left(E_{q}\right) \approx \frac{e m d_{E} \exp \left[-c_{E}\left(E_{q}\right)\right]}{2 \pi^{2} \hbar^{3}} n_{\mathrm{FD}}\left(E_{q}\right) \times \\
& \times\left\{\begin{array}{l}
1-\exp \left[-\frac{E_{q}+\varepsilon_{F}+\delta R}{d_{E}}\right], \quad E_{q}<4 t^{\prime}-\mu-\delta R \\
\frac{1}{\pi} \arccos \left[\frac{k_{t}^{\min }}{k_{t}^{\text {max }}}\right]\left\{\exp \left[-\frac{1}{d_{E}} \frac{\left(\hbar k_{t}^{\min }\right)^{2}}{2 m}\right]-\exp \left[-\frac{E_{q}+\varepsilon_{F}+\delta R}{d_{E}}\right]\right\}, E_{q}>4 t^{\prime}-\mu-\delta R
\end{array}\right.
\end{aligned}
$$

where $k_{\mathrm{t}}^{\max }=\left(\frac{2 m}{\hbar^{2}}\left(E_{q}+\varepsilon_{F}+\delta R\right)\right)^{-1 / 2}, k_{\mathrm{t}}^{\min }=\frac{1}{a} \arccos \left[-\frac{E_{q}+\delta R+\mu-2 t}{2 t-4 t^{\prime}}\right]$.

Fig. 7 shows the emission spectra, calculated according to Eq. (9) (curve 2) and by means of numerical integration of Eq. (4) (curve 1). As seen from the picture, the approxiame analytical expression (9) serves fairly well to reflect the features of field emission spectrum obtained by more accurate integration. As this takes place, the small amplitude variations are not determinative in emission spectroscopy investigations.

\section{CONCLUSION}

Therefore, the elaborated approach to describing of HTSC materials emission characteristics has made it possible to explain almost all 'abnormal' (in terms of the Fowler-Nordheim field emission theory) experimental data on $\mathrm{YBa}_{2} \mathrm{Cu}_{3} \mathrm{O}_{6.9}$ emission spectroscopy in the [100] direction, which have earlier been obtained in [11-14,23] experimentally. Let us distinguish the three most important characteristics:

(i) The abnormally great width of the high energy edge of the field emission spectrum is due to the band structure effects in these materials, with the filling to the full of the free carrier band near the emission surface, which, in turn, is caused by the external electrical field penetration 
(ii) The shift of the field emission spectrum is caused by the electric field penetration, which is nonlinear as far as the band structure is taken into account.

(iii) The topographical features of band structure lead to the fact that the total width of field emission spectrum is determined by the width of energy band and amounts, approximately, to $2 \mathrm{eV}$ almost at any electrical field strength at which field emission current is noticeable.

\section{REFERENCES}

[1] Bednorz J.G., Muller K.A. Possible High- $T_{\mathrm{c}}$ Superconductivity in the Ba-La-Cu-0 System, Z. Phys. B. - Condensed Matter 64 (1986) 189.

[2] G..A. Mesyats, N.N. Syutkin, V.A. Ivchenko, E.F. Talantsev, J. de Phys. 49 (C6) (1988) 477.

[3] E.F. Talantsev, V.A. Ivchenko, N.N. Syutkin, J. Micromechanics and Microengineering. 3 (2) (1993) 87.

[4] G.L. Kellogg, S.S. Brenner, Appl. Phys. Lett. 51 (22) (1987) 1851.

[5] G.L. Kellogg, S.S. Brenner, J. de Phys. 49 (C6) (1988) 465.

[6] A.J. Melmed, R.D. Shull, C.K.Chiang, H.A. Fowler, Science. 239 (1988) 176.

[7] A.J. Melmed, R.D. Shull, C.K.Chiang, J. de Phys. 49 (C6) (1988) 459.

[8] R.Z. Bakhtizin, S.S. Gots, V.G. Mesyats et. al., J. de Phys. 49 (C6) (1988) 495.

[9] R.Z. Bakhtizin, V.M. Lobanov, V.G. Mesyats et. al., Fiz. Metaloved. 67 (3) (1989) 610.

[10] V.G. Mesyats, Phd thesis (1989).

[11] S.I. Shkuratov, S.N. Shilimanov, S.N. Ivanov, Surface Sci. 266 (1992) 224.

[12] S.I. Shkuratov, Surface Sci. 266 (1992) 88.

[13] S.I. Shkuratov, S.N. Shilimanov, S.N. Ivanov, Supercond. Sci. Technol.6 (1993) 520.

[14] S.I. Shkuratov, S.N. Ivanov, S.N. Shilimanov, Physica C. 213 (1993) 321.

[15] F.I. Itskovich, Soviet Phys.-JETP 23 (1996) 945.

[16] J. W. Gadzuk, Phys. Rev. 182 (2) (1969) 416.

[17] I.V. Uimanov, $48^{\text {th }}$ IFES Tech. digest (2002) - Lyon, France. 
[18] A. Modinos, Field, Thermionic and Secondary Electron Emission Spectroscopy (Plenum Press, New York, 1984)

[19] J. Schmalian, D. Pines,B. Stojkovic, Phys. Rev. Lett. 80 (1998) 3839//Preprint condmat/9804129.

[20] J. W. Gadzuk, Surface Sci. 15 (1969) 466.

[21] E.A. Litvinov, I.V. Uimanov, J. de Phys. IV 6 (C5) (1996) 65.

[22] P.W. Anderson, Science. 235 (1987) 1196.

[23] S.N. Ivanov, Phd thesis (1995). 


\section{FIGURE CAPTIONS}

Fig. 1. Schematic drawing showing the bending of a band of HTSC in a strong electric field.

Fig. 2. Schematic image of a constant energy surface in the quasi-momentum space, where $d s$ is the tangential projection onto a plane parallel to the emission surface.

Fig. 3. Relative orientation of the constant energy surfaces and the potential barrier in [100] (or [010]) direction.

Fig. 4. Tangential projections of constant energy surfaces during emission in [100] direction:

(a) the surface energy is less than $E^{\mathrm{VH}}$, (b) the surface energy is greater than $E^{\mathrm{VH}}$.

Pис. 5. The bounded surface in $\boldsymbol{k}$-space, within which are the states to contribute into the field emission in the [100] direction.

Рис. 6. Field emission spectrum behavior (a), the bending of a band (b) and the width of the high energy edge of the emission spectrum (c) during emission in the [100] direction as a function of electrical field strength $F$.

Design parameters: Thomas-Fermi radius $l_{\mathrm{TF}}=3.4 \AA$, temperature $T=115 \mathrm{~K}$, work function $\varphi=4.5 \mathrm{eV}$.

Рис. 7. Field electron emission spectrum of $\mathrm{YBa}_{2} \mathrm{Cu}_{3} \mathrm{O}_{6.9}$ in the [100] direction:

1 - calculated numerically, 2 - analytical function Eq. (9).

Design parameters: $F=4 \cdot 10^{7} \mathrm{~V} / \mathrm{cm}, \varphi=4.5 \mathrm{eV}, l_{\mathrm{TF}}=3.4 \AA$

Рис. 8. Shift of the experimental field emission spectra [23] (a) and theoretical spectra

(b) for a $\mathrm{YBa}_{2} \mathrm{Cu}_{3} \mathrm{O}_{6.9}$ emitter from the (100) plane.

Design parameters: temperature $T=115 \mathrm{~K}$, work function $\varphi=4.5 \mathrm{eV}$,

Thomas-Fermi radius $l_{\mathrm{TF}}=3.4 \stackrel{\circ}{\mathrm{A}}$. 


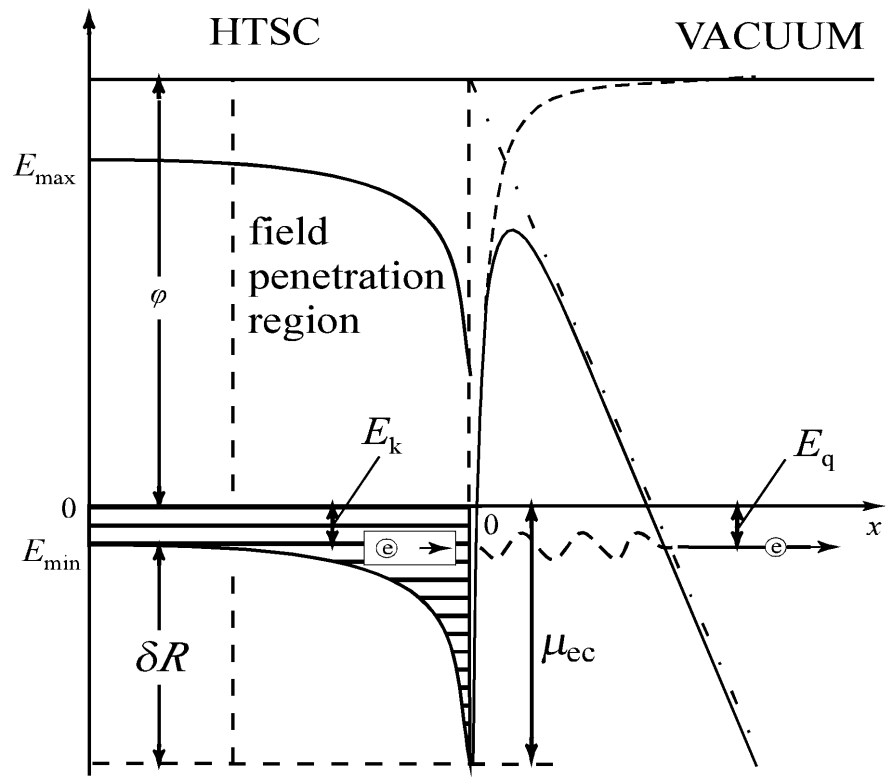

Fig. 1.

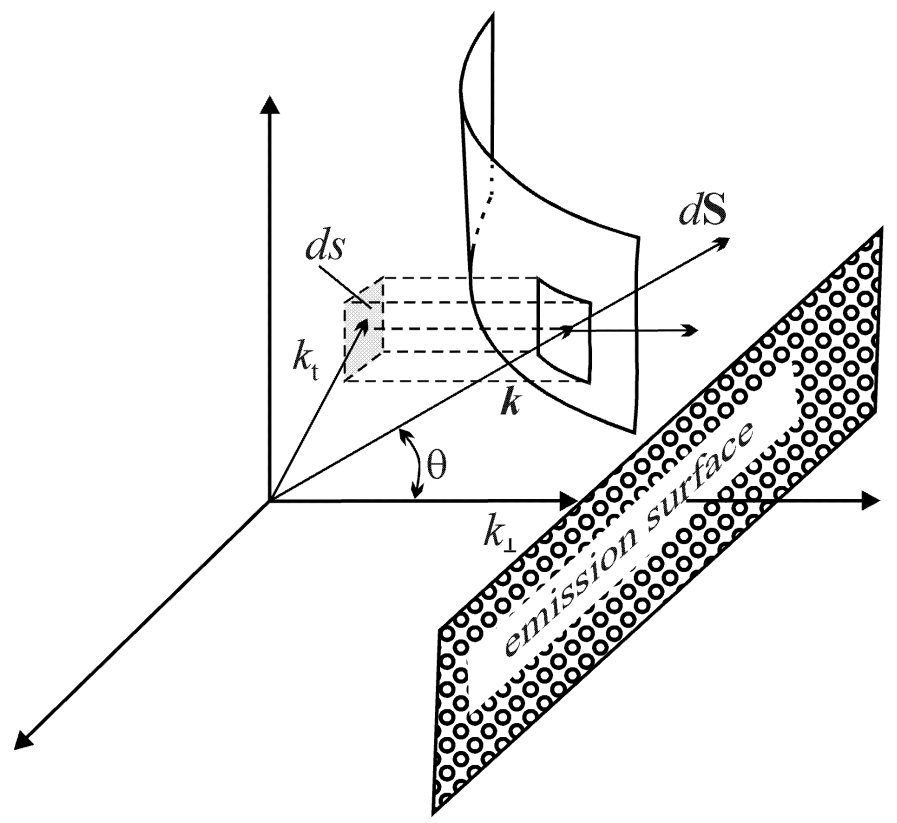

Fig. 2. 


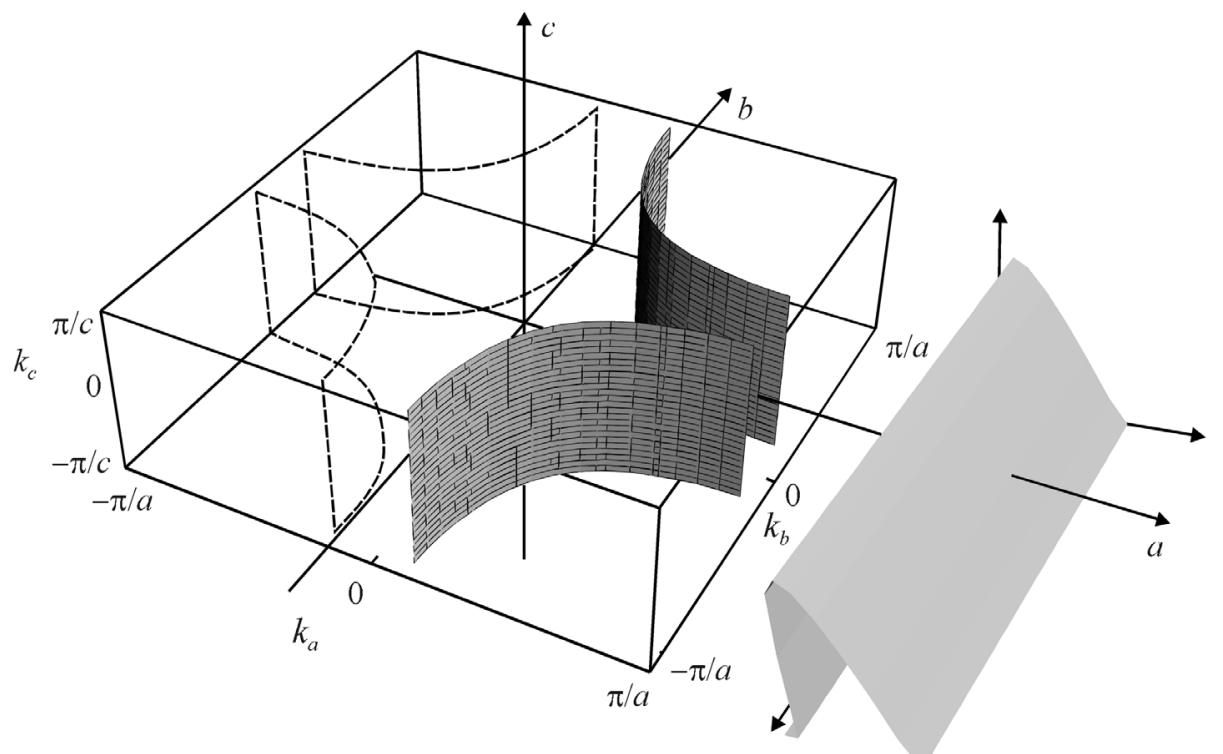

Fig. 3.
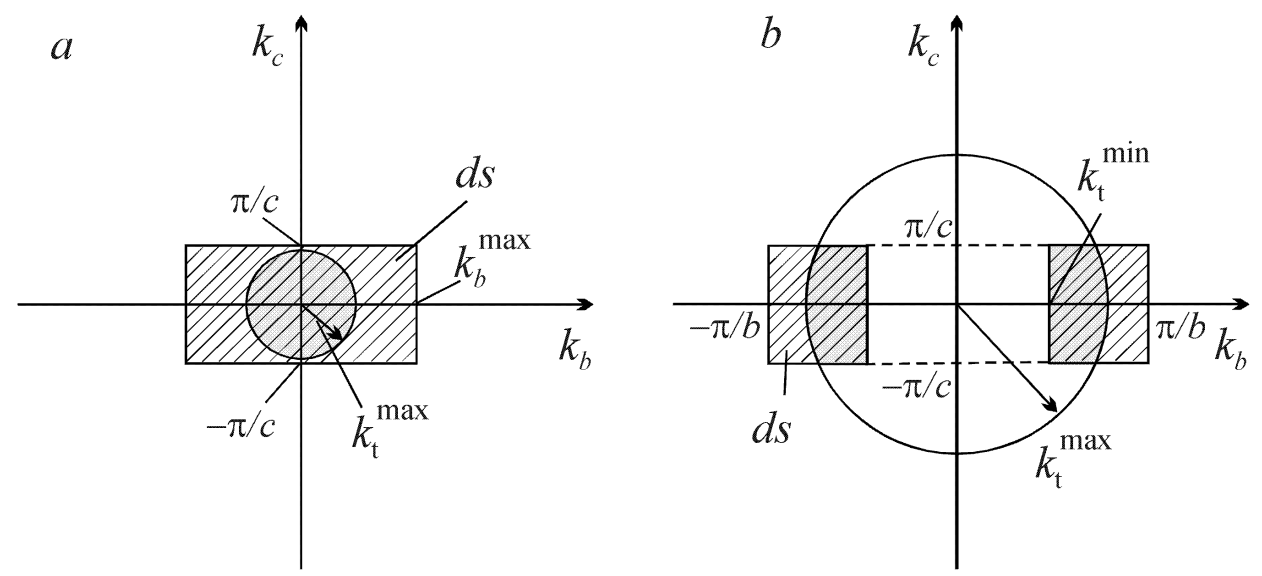

Fig. 4 a, b. 


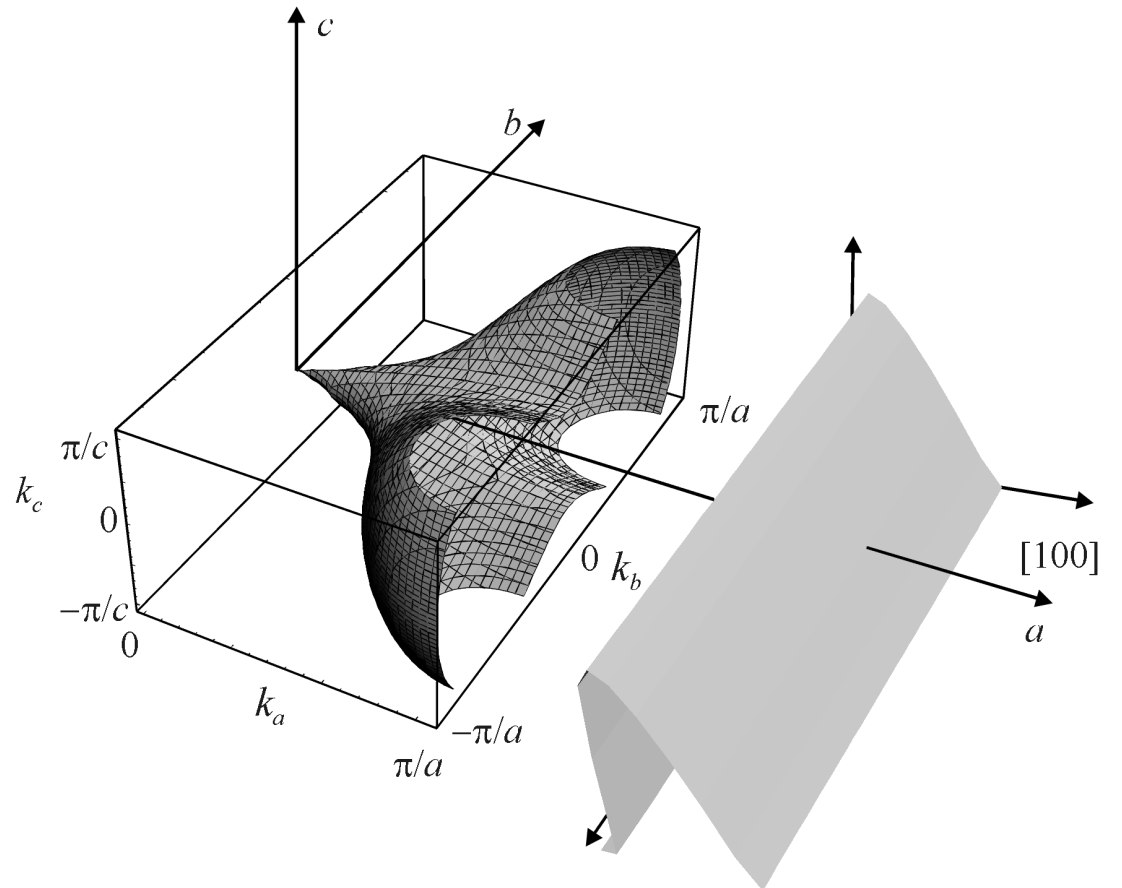

Fig. 5. 


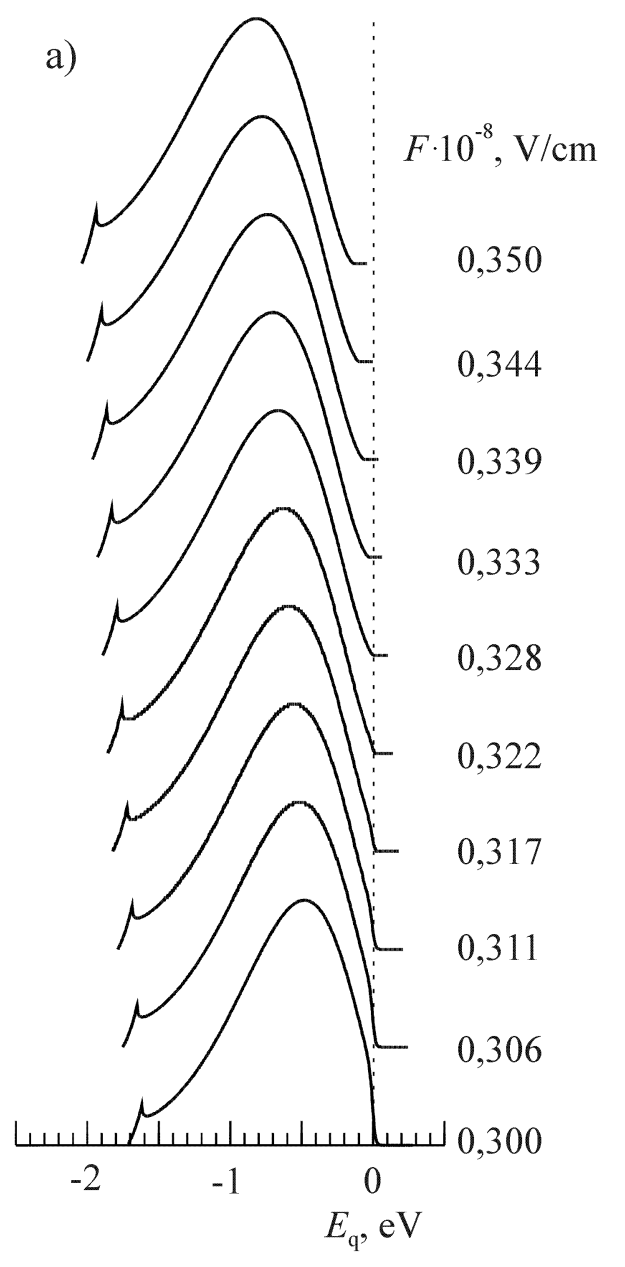

b)

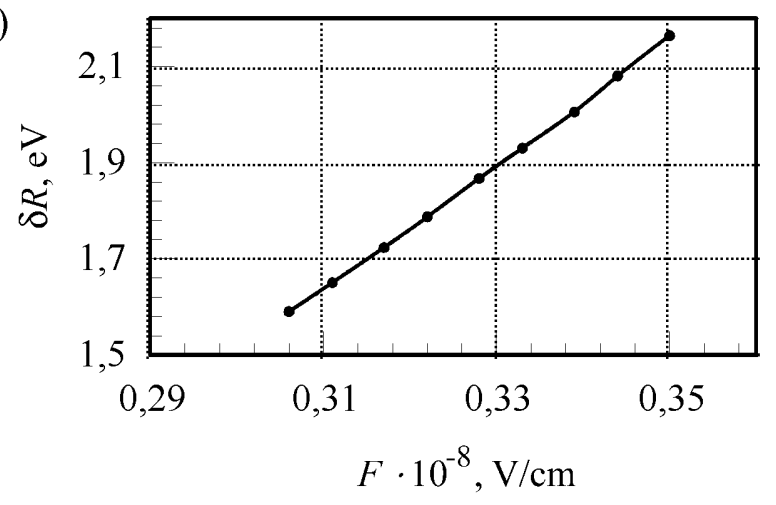

c)

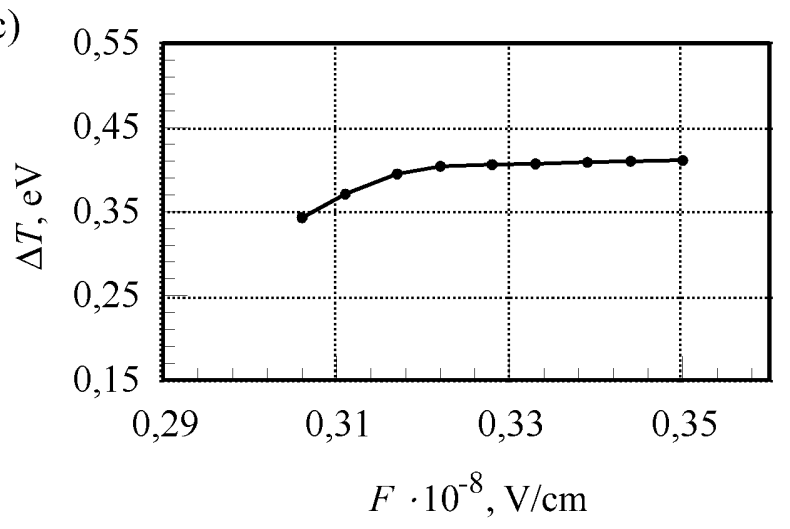

Fig. 6 a, b, c.

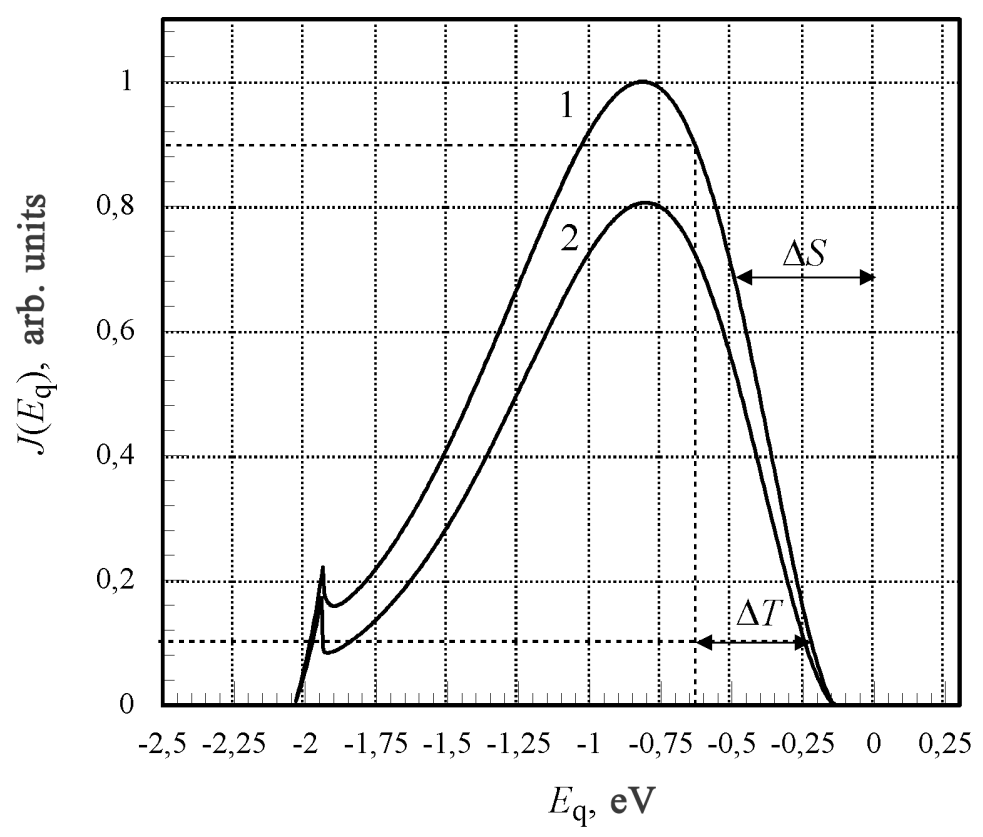

Fig. 7. 

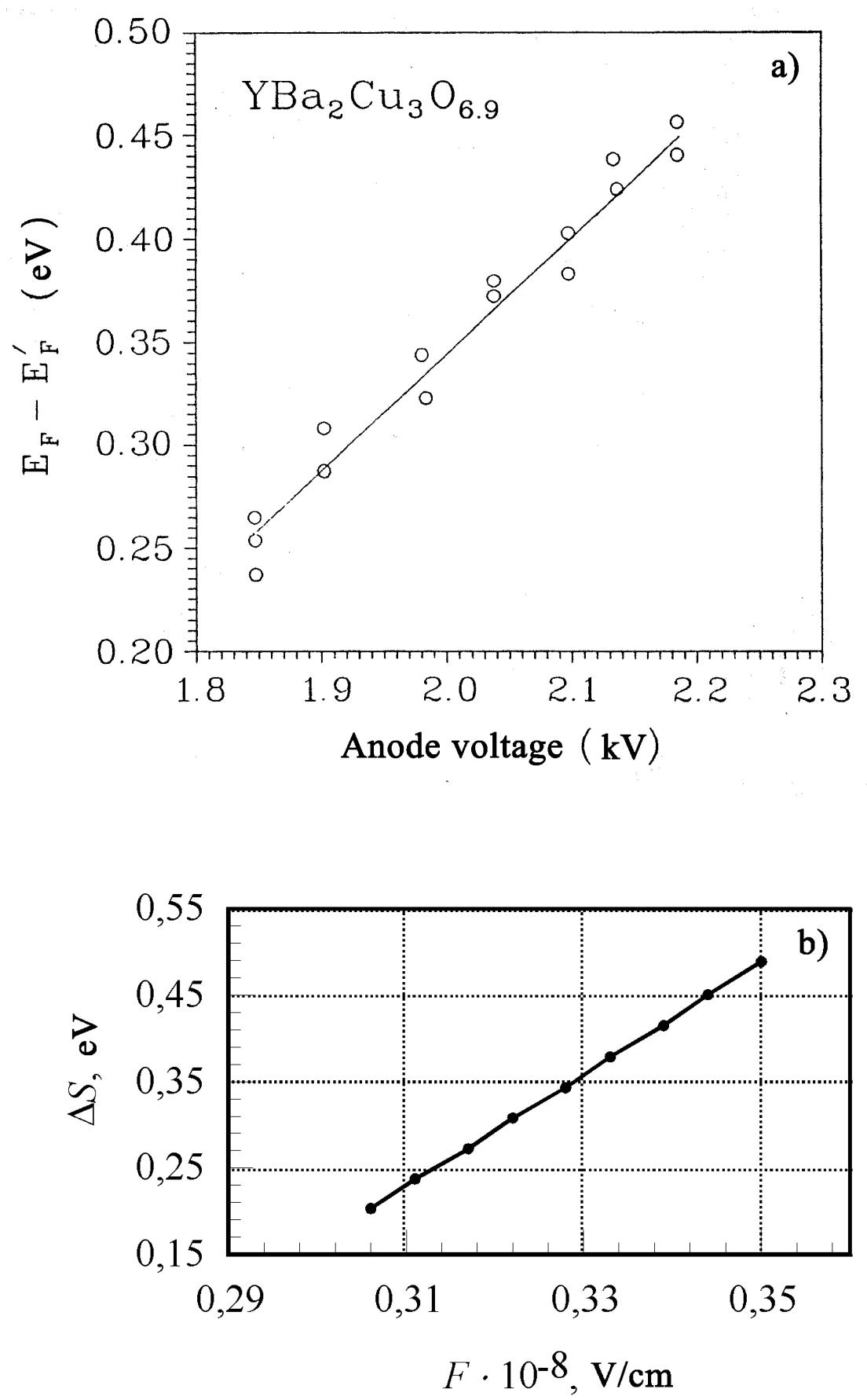

Fig. 8 a, b. 